\title{
MATHEMATICAL MODEL OF THE POPULATION DYNAMICS OF SEA TURTLES AT CHAGAR HUTANG BEACH, MALAYSIA
}

\author{
MUHAMMAD AFIQ AHMAD KHAIRUDDIN*1, UMMU ATIQAH MOHD ROSLAN ${ }^{2}$, \\ HAMIZAH MOHD SAFUAN ${ }^{3}$ AND MOHD UZAIR RUSLI ${ }^{4}$
}

${ }^{1}$ No. 8, Jalan Toh Muda Abdul Aziz, Kampung Kuala Pari, Menglembu Perak 31450 Malaysia. ${ }^{2}$ Faculty of Ocean Engineering Technology and Informatics, ${ }^{4}$ Institute of Oceanography and Environment, Universiti Malaysia Terengganu, Kuala Nerus, 21030 Terengganu Malaysia. ${ }^{3}$ Fakulti Sains Gunaan dan Teknologi, Universiti Tun Hussein Onn Malaysia, Batu Pahat, Johor Malaysia.

Universiti Malaysia Terengganu, Kuala Nerus, 21030 Terengganu Malaysia.

*Corresponding author: afiqahmadkhairuddin@gmail.com

Submitted final draft: 1 July $2020 \quad$ Accepted: 5 August 2020

http://doi.org/10.46754/jssm.2021.06.024

\begin{abstract}
In this paper, we explore the existing prey-predator dynamics between the sea turtle and its predator within a secluded bay known as Chagar Hutang on Redang Island, off the coast of Terengganu state on Peninsular Malaysia's east coast. Previous studies show that the extinction of the sea turtle is imminent and there is ample evidence of the decreasing sea turtle population in Malaysia. The authors introduce a new mathematical model of an ordinary differential equation (ODE) as a conservation initiative formulated from the literature of previous studies. This paper also presents a qualitative analysis, that is the stability analysis such as the Jacobian matrix and its eigenvalues. The authors find that the population of sea turtle eggs, hatchlings and the predators in Chagar Hutang may reach either total extinction or co-existence in a stable state in specific conditions. With the specific condition obtained, the model is simulated and the time series plot with the phase portrait diagram is further discussed by the authors.
\end{abstract}

Keywords: Green turtle, conservation, stability analysis, ordinary differential equation, dynamical system, stage-structured prey-predator model

\section{Introduction}

Predation in the wild is intense but vital to ecosystem equilibrium. But some species face total extinction due to human intervention or climate change as described by Hoppensteadt (2006). Scientists are obliged to conserve, perhaps preserve some of the endangered species by balancing the prey and predator relationship, as discussed by Ackerman (1980) of the target species, which is the sea turtle. There are seven species of sea turtle found along coasts and in the ocean. Six out of seven of them are either on the vulnerable or endangered IUCN Red List, due to the loss of habitat and spawning grounds, as well as predation by other species, Predators include ghost crabs (eggs or juvenile) onshore or in the ocean by black-tipped shark, which is considered as a specialized hunter of amphibious prey, particularly sea turtle, as described by Fitzpatrick, Thums, Bell, Meekan, Stevens and
Barnett (2012) via the predator's movement pattern on one of the common species nesting at Chagar Hutang turtle sanctuary. The green (Chelonia mydas) and hawksbill (Eretmochelys imbricata) turtles are the focal species for conservation of Sea Turtle Research Unit (SEATRU), a subsidiary unit of the Institute of Oceanography and Environment, Universiti Malaysia Terengganu. Most of the species reach maturity by 20 years, and will come ashore to nest. Approximately, 65 to 100 eggs are deposited per nest, while some species can lay up to 180 eggs (Bolten, 2002). The location of sea turtle hatchlings is barely known until they mature. The time between hatching and nesting for the first time is considered as 'lost years' of the life cycle of the sea turtle. However Bolton (2002) has highlighted three parts of the general life cycle of sea turtle: neritic phase, neriticoceanic phase, and fully oceanic phase. This life cycle is significant to the amount and types 
of predators they encounter before they reach maturity.

There are two main methods to see species interaction, either a stochastic approach or a deterministic approach that follows a certain time-dependent set of rules known as the dynamical system (Meiss, 2007). This system could describe a mathematical model of the interaction intuitively by aesthetic, qualitative measures, which is called compartmental diagram. Jaquez and Simon (1993) further explain that the method is pragmatic to construct by logical means of ecological interaction, physical phenomenon and many more provided that the diagram satisfies the conditions from the paper.

One of the pioneers of mathematical modeling of population dynamics is Pierre Francois Verhulst. He worked out an equation based on a simple population process for yielding the sustainable yet maximizing capacity of the population. Initially, it was seen as a Malthusian population growth model in 1798. But the model behaves like a suppressed exponential graph at one part and then plateaus when reaching a point, named logistic graph (Bacaër, 2011). Nearly a century later, Raymond Pearl and Lowell Reed refined the finding. This Pearl-Verhulst Model, which is known as a population growth model, introduced the term "carrying capacity" and was supported by another model called the LotkaVolterra equation, originally used by A.J Lotka in autocatalytic chemical reaction phenomenon and later to prey-predator interactions, which was at the same time Vito Volterra's observation on fishery activity post-World War 1 (Canale, 1970 \& Bacaër, 2011).

Stability analysis is one of the many solution methods for the prey-predator model, as well as a key point on measuring sustainability for conservation effort for the subject of interest. The stability of equilibria of this model can be obtained via solving the Ordinary Differential Equation (ODE) system, which is a system of derivative equations, by using Jacobian matrices and obtaining the eigenvalues of the matrices by solving the matrices. Phase portrait, on the other hand, deals with patterns of interest between the predators towards different values of prey, projected on flows of changes in a graph, in most cases in 2 dimensions ( $\mathrm{Hu} \&$ Cao, 2017; Kant \& Kumar, 2016; Lynch, 2014). Various applications of the model emerge, such as a Lotka-Volterra model on a general prey-predator system via stability analysis and 2D phase portrait of the interaction by Lynch (2014), complex Lotka-Volterra model with Holling type II systems as proposed by Liu and Chen (2003), as well as migrating prey-predator model towards secluded sanctuary with disease factors (Lakshmi, 2003) and the global stability and bifurcation analysis of the stage-structured prey-predator system, as thoroughly discussed by Chakraborty et al. (2012).

Researchers show their enthusiasm towards the effectiveness of this model by running it on time series analysis. It is rather costly and timeconsuming to have sea turtle as subject to study in a short span of time due to the longevity of this species, thus the approach of modelling the population projection is a must and comparing them to seek the effectiveness of the SEATRU project in Chagar Hutang has been a concern. By using mathematical modelling, specifically time-series analysis of the model, we can estimate the impact of the program for 10 to 20 years to come. Recently, a first ODE model describing the sea turtle-human interaction was introduced by Mohd Roslan et al. (2019). In the paper, the effect of changing consumption rate on the population of sea turtles was investigated via stability analysis method. Their findings show that the sea turtle population can be sustained if there is no intervention from humans.

In this paper, a stage-structured turtle population model coupled with Lotka-Volterra predation dynamics for describing sea turtle sustainability in Chagar Hutang by analysing the dynamics of the population of the juveniles as well as the predator that lives symbiotically with them, and also evaluating the effectiveness of the sea turtle sanctuary for many years to come. From the developed model, we investigate the stability of equilibria values of the three by using 
a method called the stability analysis. Based on the results, we then find the projection of the optimized parameter to the maturity period of the batch, and plot the population over time by the method of time series.

\section{Model Assumption and Formulation}

There are some assumptions for the model to work properly. First, the predator will feed exclusively on the hatchlings. So, when the prey population depletes, the predator will be significantly impacted. Second, there will be no intervention from external factors, such as mass extinction due natural disaster, etc. Third, the predator will never have chances to feed on the same prey if unsuccessful, and returns to the pool of prey and this assumption relates to the likelihood of encountering the same prey at sea for a short span of time is quite low due to the vastness of the sea. Fourth, the predator described in this paper is a general predator of coastal and sea turtles. The parameter $w$ is the combined predation rate of all possible predators and variable $z$ is the general population of all predators possible that attack the hatchlings. The final inference is that the sea turtle is oviparous, and there are some stage-structured growth mechanisms, from the eggs in the nest to the hatchlings to adulthood, which are similar to other animals like moths and flies.

The Lotka-Volterra prey predator system traditionally consists of two equations that model the interaction between a prey (denoted by $x$ ) and predator (denoted by $y$ ). The original Lotka-Volterra model (Volterra, 1926) is given by

$$
x=\frac{d x}{d t}=\alpha x-\beta x y, y=\frac{d y}{d t}=\delta x y-\gamma y,
$$

where $\alpha, \beta, \delta, \gamma$ are positive real parameters which represent the growth rate of the prey, predation rate by the predator, conversion rate of prey to predator and natural death rate of the predator, respectively. Our new model is constructed based on this model. In our case, we extend the above model to three equations, where the prey is divided into two stages, ie the sea turtle eggs (denoted by $x$ ), the sea turtle hatchlings (denoted by $y$ ) and the marine predator, such as sharks, barracuda and crabs (denoted by $z$ ). Thus, we introduce the following stage-structured preypredator ODE model as

$$
\begin{gathered}
x=\frac{d x}{d t}=r x\left(1-\frac{x}{K}\right)-a x-w x, y=\frac{d y}{d t}=g w x-c y-b y z, z \\
=\frac{d z}{d t}=f b y z-s z \\
\quad x(0) \geq 0, y(0) \geq 0, z(0) \geq 0,
\end{gathered}
$$

where the variables and the parameters of model (1) are described in Table 1, intuitively as described by May and McLean (2007) for the selection of suitable variables of sea turtle populations. For the proof that a uniform boundary exists in the system (1), the steps are from Das and Pal (2019).

The nonlinear term $r x\left(1-\frac{x}{K}\right)$ means that the growth rate $r$ of the sea turtle eggs follows the logistic type, with carrying capacity $K$, where $K$ represents the maximum number of eggs that can occupy the nest at any time. The terms $a x$, $w x$ and $c y$ represent the natural infertile rate of the eggs, stage conversion rate of the eggs to successfully hatched and natural death rate of the hatchling respectively. The term $g$ in $g w x$ is the effective phase change from hatched eggs to juvenile. Meanwhile, the term byz represents the decreased population of hatchlings due to predation. On the other hand, the predator of the hatchlings gains its population by converting the predation rate to reproduction rate by the term $f b y z$, and $s z$ is the natural death rate of the predator.

For the system to be biologically meaningful, the solutions of the system must always be positive and bounded. To establish 
Table 1: Variables and parameter description for model (1)

\begin{tabular}{|c|c|c|c|}
\hline Variables & Description & Values & References \\
\hline$x$ & $\begin{array}{l}\text { The number of sea turtle eggs that is } \\
\text { successfully incubated }\end{array}$ & - & Unknown \\
\hline$y$ & The number of hatchlings & - & Unknown \\
\hline$z$ & The number of marine predator & - & Unknown \\
\hline Parameters & Description & Values & References \\
\hline$r$ & $\begin{array}{l}\text { Rate of eggs laid in the nest by adult } \\
\text { sea turtle }\end{array}$ & 1.3 & Bjorndal et al., 2000 \\
\hline$K$ & $\begin{array}{l}\text { Carrying capacity for egg nesting per } \\
\text { year }\end{array}$ & 700 & Bjorndal et al., 2000 \\
\hline$a$ & $\begin{array}{c}\text { Rate of eggs that fail to hatch (natural } \\
\text { death) }\end{array}$ & 0.2 & Wyneken et al., 1988 \\
\hline$w$ & Rate of eggs that hatch & 0.53 & Abella et al., 2017 \\
\hline$c$ & $\begin{array}{l}\text { Survival rate (chances of juveniles } \\
\text { surviving predation) }\end{array}$ & 0.001 & IAC, 2004 \\
\hline$b$ & $\begin{array}{l}\text { Predation rate for newly hatched sea } \\
\text { turtle juveniles }\end{array}$ & 0.015 & Gyuris, 1994 \\
\hline$g$ & $\begin{array}{l}\text { Conversion rate for newly hatched sea } \\
\text { turtle juveniles }\end{array}$ & 1 & Gyuris, 1994 \\
\hline$s$ & Predator natural death rate & 0.7 & Assumed \\
\hline$f$ & $\begin{array}{c}\text { Conversion rate of prey to predator via } \\
\text { predation }\end{array}$ & 1 & Assumed \\
\hline
\end{tabular}

the proof of positivity of solutions, we need to rearrange equation (1) as follows:

$$
\begin{gathered}
\frac{d x}{x}=\left(r\left(1-\frac{x}{K}\right)-a-w\right) d t \frac{d y}{y}=\left(\frac{g w x}{y}-(c+b z)\right) d t \frac{d z}{z} \\
=(f b y-s) d t
\end{gathered}
$$

By integrating both sides of the above equations, we obtain the solutions as follow:

$$
\begin{array}{ccc}
x(t)=x(0) \exp \left(\int \quad r\left(1-\frac{x}{K}\right)-a-w d t\right)(\geq 0) & y(t) \\
=y(0) \exp \left(\int \frac{g w x}{y}-(c+b z) d t\right) & (\geq 0) z(t) \\
=z(0) \exp \left(\int f b y-s d t\right) & (\geq 0)
\end{array}
$$

Thus the solution will always be positive. 
To prove the boundedness of the system, left and all positive terms on right as shown in we must first collect the negative terms on the equation (4).

$$
\begin{gathered}
\frac{d x}{d t}+(a+w) x \leq r x\left(1-\frac{x}{K}\right) \\
\frac{d y}{d t}+(c+b z) y \leq g w x \\
\frac{d z}{d t}+s z \leq f b y z
\end{gathered}
$$

Establish the possible maximum value of $x$, $y$ and $z$ respectively in (5),

$$
\begin{gathered}
\text { let } x=r x\left(1-\frac{x}{K}\right), y=g w x, z=f b y z \\
x=\frac{r K}{4}, y=g w\left(\frac{r K}{4}\right), z=\left(f b g w\left(\frac{r K}{4}\right)\right)^{3}
\end{gathered}
$$

Next, the maximum value of $x, y$ and $z$ in (5) is plugged into (4) and then solved using integrating factors to obtain (6) combine all terms in (6) and plug in the maximum value obtained in (4) and thus the boundedness of the solution of model (1) is proved in equation (7).

$$
\begin{gathered}
\text { let } e^{\int(a+w)^{t}}=e^{(a+w) t}, e^{\int(c+b z) t}=e^{(c+b z) t}, e^{\int(s)^{t}}=e^{(s)^{t}} \\
e^{(a+w) t}\left[\frac{d x}{d t}+(a+w)^{x}\right] \leq \frac{r K}{4} e^{(a+w)^{t},} \\
e^{(c+b z) t}\left[\frac{d y}{d t}+(c+b z) y \mid \leq g w\left(\frac{r K}{4}\right) e^{(a+w)^{t}},\right. \\
e^{(s)^{t}}\left|\frac{d z}{d t}+(S)^{z}\right| \leq\left(f b g w\left(\frac{r K}{4}\right)\right) e^{(s)^{t}} .
\end{gathered}
$$

$$
\begin{gathered}
\left\{x, y, z \in R^{3+}, 0<x, y, z \leq L_{1}, L_{2}, L_{3}\right\} \\
L_{1}=\frac{r K}{4(a+w)}, L_{2}=\frac{g w\left(\frac{r K}{4}\right)}{c+b z}, L_{3}=\frac{\left(f b g w\left(\frac{r K}{4}\right)\right)^{3}}{s}
\end{gathered}
$$

\section{Materials and Methods}

\section{General Framework of Stability Analysis}

One of the important analysis in dynamical system is the stability analysis, in this context is the stability of the three species in system (1). From the previous section, we have computed for the equilibrium points, i.e. the equilibria. Biologically, this equilibria means that we are finding the possible solutions (i.e. the number of the three species) in which we anticipate to occur in the future. Generally, there will be more than one possible solution for a dynamical system. Thus, in order to predict which solution manifests in the future, we employ the method of stability analysis. If the equilibrium point is stable, then the corresponding equilibrium will happen in the future. Meanwhile, if the equilibrium point is not stable, this equilibrium will not happen in the future.

There are steps to be taken to obtain equilibria and stability values. The steps described below are based on Mohd Roslan et al. (2019):

Step 1: Find the equilibrium values (also known as the number of eggs, hatchlings and marine predators' population) by setting the equations of the model (1) to 0 , and solve them simultaneously. 


$$
r x\left(1-\frac{x}{K}\right)-a x-w x=0 g w x-c y-b y z=0 f b y z-s z=0
$$

Step 2: Obtain the general Jacobian matrix for the model (1), where this will linear system by linearization method. transform the nonlinear model into a

$$
J(x, y, z)=\left(\frac{\partial x}{\partial x} \frac{\partial x}{\partial y} \frac{\partial x}{\partial z} \frac{\partial y}{\partial x} \frac{\partial y}{\partial y} \frac{\partial y}{\partial z} \frac{\partial z}{\partial x} \frac{\partial z}{\partial y} \frac{\partial z}{\partial z}\right)
$$

Step 3: The general Jacobian matrix that consists of only the variables and parameters of interest is obtained as follows.

$$
\begin{gathered}
J(x, y, z)=\left(-(a+w) r\left(1-\frac{y}{K}\right)-\frac{r y}{K} 0 g w-b z-c-\right. \\
\text { by } 0 z f b \text { bf } y-s) .
\end{gathered}
$$

Step 4: We then substitute equilibria obtained in Step 1 into matrix (10). Note that for $n$ equilibrium, value will yield $n$ Jacobian matrices.

Step 5: Determine the eigenvalues for each of the Jacobian matrices by solving $\operatorname{det}|\lambda-I J|=0$, where $\lambda$ represents the eigenvalues, $I$ is for the identity matrix and $J$ is for the Jacobian matrix in equation (6).

Step 6: The final step is determining the stability of the equilibrium points by observing whether the sign of the eigenvalues is positive or negative.

\section{Results and Discussion}

Some possible points of equilibria obtained in Step 1 after solving the equation (2) with simultaneous equation operation are the trivial solution

$$
\begin{aligned}
& E_{0}\left(x^{*}, y^{*}, z^{*}\right)=(0,0,0), \\
& E_{1}\left(x^{*}, y^{*}, 0\right)=\left(\frac{K c(g r w+c w-a c)}{g^{2} r w^{2}}, \frac{K c(g r w+c w-a c)}{g r w}, 0\right)
\end{aligned}
$$

which represents the absence of predators in the system and,

$$
E_{2}\left(x^{*}, y^{*}, z^{*}\right)=\left(\frac{r s(K b f-s)}{b^{2} f^{2} K(a+w)}, \frac{s}{b f}, \frac{K b f g r w-K a b c f-K b c f w-g r s w}{K b^{2} f(a+w)}\right)
$$

which represents the co-existence of eggs, juveniles and predators. Since $E_{0}$ is just trivial, we ignore this equilibrium point. To complete the results in Steps 3-5, each equilibrium point above is then plugged into equation (4) to find general eigenvalue. For the solution to be asymptotically stable, the eigenvalue must always be negative-definite. The summary of the equilibria with its positivity condition and the characteristic polynomial at every possible equilibrium points are shown in Table 2.

By plugging in the values from Table 1 into Table 2, we can obtain the eigenvalues by finding the roots of the characteristic equation that is shown in Table 3 below. For the model with no predator present, one of the real parts of the eigenvalue is positive, thus the condition is not stable, whereas in the coexistence model, all of the real parts of the eigenvalues are negative, thus we conclude the model to be stable. Note that there is a mix of stable and unstable foci by the negative (stable) and positive (unstable) of the complex part of the eigenvalues of $E_{1}$ and $E_{2}$. This concludes the solution for finding a generalization of eigenvalues and the equilibria of equation (1). 
Table 2: Equilibria and its condition with the Characteristic Polynomial

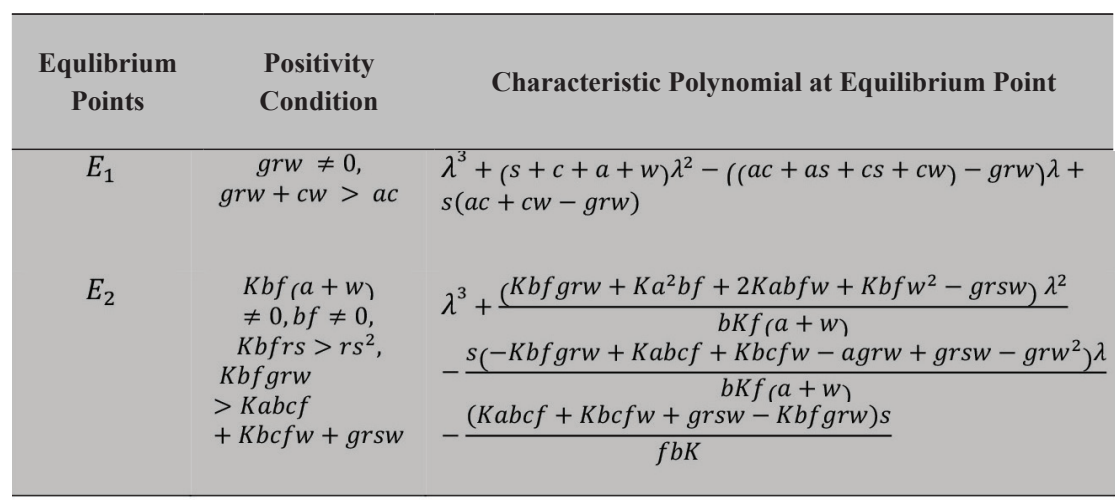

Table 3: The eigenvalues and the stability types for each equilibrium point

\begin{tabular}{cccc}
$\begin{array}{c}\text { Equilibrium } \\
\text { Points }\end{array}$ & Characteristic Equation & Eigenvalues & $\begin{array}{c}\text { Stability } \\
\text { Types }\end{array}$ \\
\hline$E_{1}$ & $\lambda^{3}-8.94875 \lambda^{2}-6.48798 \lambda$ & $\begin{array}{c}\lambda_{1}=9.68875 \\
\lambda_{2}=-0.37+0.7381 i\end{array}$ & Unstable \\
& & $\lambda_{3}=-0.37-0.7381 i$ & \\
\hline \multirow{2}{*}{$E_{2}$} & $\lambda^{3}+1.6109 \lambda^{3}+0.6555 \lambda+0.44503$ & $\lambda_{1}=-1.3695$ & Stable \\
& & $\begin{array}{l}\lambda_{2}=-0.12+0.5571 i \\
\lambda_{3}=-0.12-0.5571 i\end{array}$ & \\
\hline
\end{tabular}

From the results of steps 1 to 5 in the methodology section, all possible variables and parameter values in Table (1) in the previous sections assimilated into model (2), as shown in the simplified version in the work of Crouse et al. (1987). Turtle eggs are part of the food chain, the sea turtle embryos absorb nutrients in the eggs, and develop and transform into hatchlings. To simulate the statement above, we used the Maple software to obtain the following figures. For the initial value, the number of eggs is 1,000 , the number of sea turtle hatchlings is set at 100 and the number of predators is 10 . The algorithm used in Maple to solve the ODE model is the default settings of Initial Value Problem (IVP), that is the Runge-Kutta Fehlberg method.

From Figure 1, it is observed that if the sea turtle conservation project in Chagar Hutang is prolonged by 50 years, we can see that in the first 10 years, the population will achieve maximum population capacity and slowly decrease at the 10 to 20 year mark and eventually plateau. Theoretically, according to May and McLean (2007), the point in the graph when prey and predator intersect is when the populations reach
Green (sea turtle eggs), Blue (sea turtle hatchlings) and Red (predator)

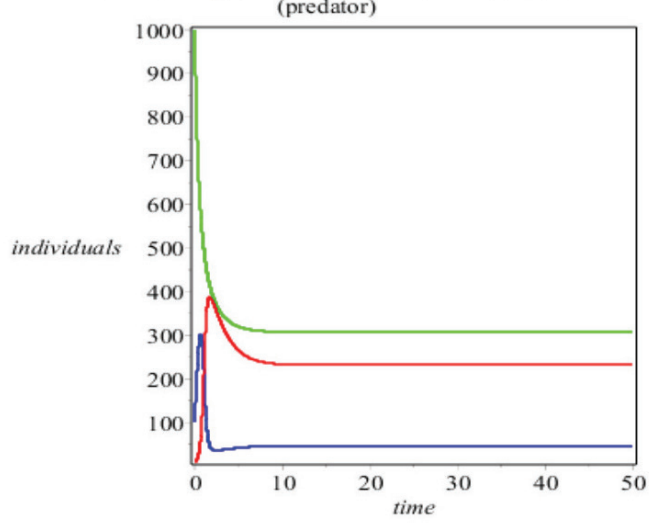

Figure 1: Time series diagram for $t=0$ to $t=50$ where blue represents eggs, green represents sea turtle hatchlings and red is the predator population. This image is generated using the Maple software

equilibrium, which can be observed at the first 10 years of the program. However, all variables are expected to co-exist provided that the parameter is not modified.

In Figure 2, the left and right phase diagram plots are not identical, and there exists 

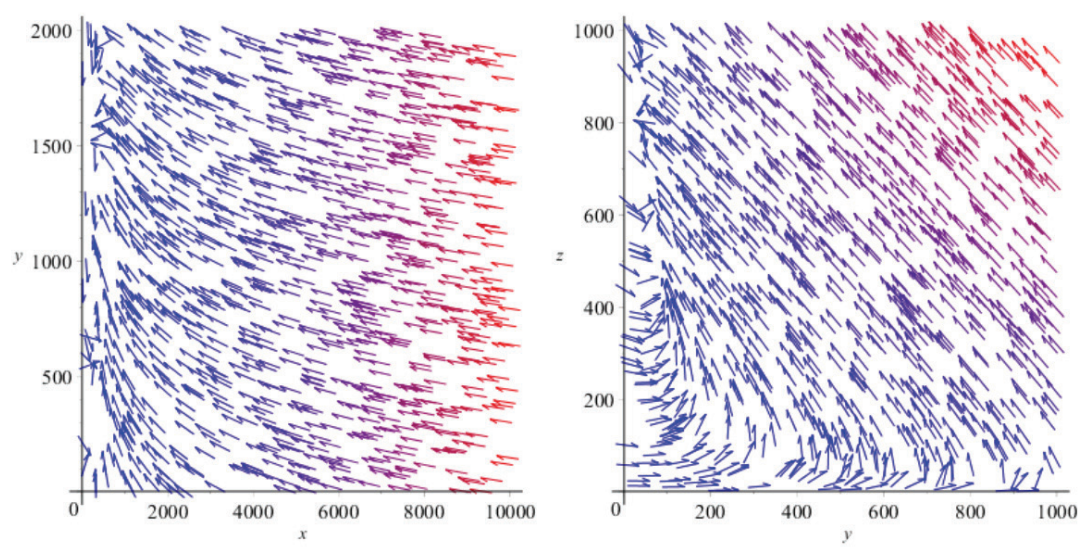

Figure 2: Phase portrait diagram of eggs against sea turtle hatchling (left) and sea turtle hatchling against predator (right). This image is generated using the Maple software

a set of complex nullclines in both diagrams. In the right diagram of Figure 2, the trajectories of the eggs against the hatchling population tend to converge at the range of 100 eggs and 400 hatchlings for the lower bound and 50 eggs and 900 for the upper bound, marking a narrow region of convergence. The same narrow convergence pattern is also observed at the left diagram in the region of 50-100 hatchlings to $400-900$ predators in Figure 2. There is no visible limit cycle found and there is no single convergence point in the left and right diagrams of Figure 2.

\section{Conclusion}

In this paper, the authors have modelled the general dynamics of the population of a stagestructured sea turtle population from the eggs to the juvenile sea turtle spanning 50 years of marine predator population interaction at Chagar Hutang beach, Terengganu. From Table 3, we conclude that predators play an important role in maintaining stability of the sea turtle population at Chagar Hutang beach. Assuming that this research continues for 50 years, we conclude that the conservation program will achieve its maturity as early as the 10th year. If there are no significant changes to the parameters, the system will stay at equilibrium forever. This will be the ultimate goal of the conservation effort of SEATRU UMT at Chagar Hutang, and perhaps be a leading example of sustainable conservation programmes for endangered species, specifically green turtles. However, there is a limitation to this model, as it does not consider the nature of predation, which is the eat-process-eat cycle, which is the functional response between the prey and predator, because this will affect the prey-predator dynamics as whole and is more realistic compared to classical Lotka-Volterra prey predator systems. Furthermore, the parameters may be tested through bifurcation of the model to find out whether the system will maintain the stability or not. For further research, the off-season pattern and the sea turtle nesting behaviour in nesting seasons should also be considered into the model, as these factors are estimated to have a significant effect on the system. Another suggestion for further research is to compare the real data with this deterministic model in order to evaluate its efficiency and accuracy.

\section{Acknowledgements}

This work is funded by the Fundamental Research Grant Scheme (FRGS) FRGS/1/2019/ STG06/UMT/03/1 awarded under the Malaysia Ministry of Education. The authors acknowledge the turtle rangers, volunteers and fellow researchers at Marine Turtle Field Station of Chagar Hutang for their assistance in data collection. The authors also thank the referees for providing very helpful comments and advice. 


\section{References}

Abella Pérez, E., García Cerdá, R. M., \& Marco, A. (2017). Estimating the fertilization rate of sea turtle nests: Comparison of two techniques. Basic and Applied Herpetology.

Ackerman, R. A. (1980). Physiological and ecological aspects of gas exchange by sea turtle eggs. American Zoologist, 20 (3), 575583. https://www.jstor.org/stable/3882486

Bacaër, N.(2011). Ashorthistory of mathematical population dynamics. Springer - Verlag.

Banerjee, M. (2016). Solution for systems of linear ordinary differential equations - Phase portraits. https://youtu.be/ SHS4zsNu8y8

Bjorndal, K. A., Bolten, A. B., \& Chaloupka, M. Y. (2000). Green turtle somatic growth model: Evidence for density dependence. Ecological Applications, 10(1), 269-282.

Bolten, A. B. (2002) Variation in sea turtle life history patterns: Neritic vs. oceanic developmental stages. The Biology of Sea Turtles, 2, 243-257.

Canale, R. P. (1970). An analysis of models describing predator-prey interaction. Biotechnology and Bioengineering, 7, 353378.

Chakraborty, K., Jana, S., \& Kar, T. K. (2012). Global dynamics and bifurcation in a stage structured prey-predator fishery model with harvesting. Applied Mathematics and Computation, 218(18), 9271-9290. https:// doi.org/10.1016/j.amc.2012.03.005

Crouse, D. T., Crowder, L. B., \& Caswell, H. (1987). A stage-based population model for loggerhead sea turtles and implications for conservation, 68(5), 1412-1423. http:// www.jstor.org/stable/1939225

Fitzpatrick, R., Thums, M., Bell, I., Meekan, M. G., Stevens, J. D., \& Barnett, A. (2012). A comparison of the seasonal movements of tiger sharks and green turtles provides insight into their predator-prey relationship. PloS One, 7(12), e51927. https://doi.org/10.1371/journal.pone. 0051927

Gyuris, E. (1994). The rate of predation by fishes on hatchlings of the green turtle (Chelonia mydas). Coral Reefs, 13(3), 137-144.

Hoppensteadt, F. (2006). Predator-prey model. Scholarpedia, 1(10), 1563. https://doi. org/10.4249/scholarpedia.1563

Hu, D., \& Cao, H. (2017). Stability and bifurcation analysis in a predator-prey system with Michaelis- Menten type predator harvesting. Nonlinear Analysis: Real World Applications, 33, 58-82.

IAC Secretariat (2004). An Introduction to sea turtles of the world.

Jacquez, J. A., \& Simon, C. P. (1993). Qualitative theory of compartmental systems. SIAM Review, 35(1), 43-79.

Kant, S., \& Kumar, V. (2016). Dynamical behavior of a stage structured prey-predator model. International Journal of Nonlinear Analysis and Applications, 7(1), 231-241.

Lakshmi, B. S. (2003). Oscillating population models. Chaos, Solitons \& Fractals, 16(2), 183-186.

Liu, X., \& Chen, L. (2003). Complex dynamics of holling type II Lotka-Volterra predatorprey system with impulsive perturbations on the predator. Chaos, Solitons \& Fractals, 16(2), 311-320.

Lynch, S. (2014). Dynamical systems with applications using matlab. New York N.Y.: Springer Science+Business Media LLC.

May, R., \& McLean, A. R. (2007). Theoretical ecology: Principles and applications. Oxford: University Press on Demand.

Meiss, J. (2007). Dynamical systems. Scholarpedia, 2(2), 1629. https://doi. org/10.4249/scholarpedia.1629

Mohd Roslan, U. A., Safuan, H., \& Mat Piah, R. (2019). Squids-snapper fish dynamics model with fishing effects in Terengganu, 
Malaysia. Malaysian Applied Biology, 48, 235-240.

Mohd Roslan, U. A., Jailani, M. S. O., \& Rusli, M. U. (2019). Stability analysis for the dynamics of new sea turtle-human interaction model, Journal of Advanced Research in Dynamical and Control Systems, 11(12), 171-177.
Volterra, V. (1926). "Variazioni e fluttuazioni del numero d'individui in specie animali conviventi”. Mem. Acad. Lincei Roma, 2, 31-113.

Wyneken, J., Burke, T. J., Salmon, M., \& Pedersen, D. K. (1988). Egg failure in natural and relocated sea turtle nests. Journal of Herpetology, 88-96. 